\title{
The Internet of Services and USDL
}

\author{
Orestis Terzidis, ${ }^{1}$ Daniel Oberle, ${ }^{1}$ and Kay Kadner ${ }^{2}$ \\ 1 SAP Research Karlsruhe, Germany, orestis.terzidis@sap.com, \\ d.oberle@sap.com \\ 2 SAP Research Dresden, Germany, kay.kadner@sap.com
}

\section{Introduction}

The services sector is an economic growth driver in most developed economies. As an example, consider the Federal Republic of Germany, where the largest part of the macroeconomic value of 2009 is generated by the service industry [ 5 , page 637]. This trend coincides with the ongoing industrialization of the services sector in developed economies. In general, the three driving forces of every industrialization process have been automation, standardization, and specialization. First, the Information and Communications Technology (ICT) serves as an $\mathrm{Au}$ tomation and transformation factor. At the low end, automation in the services sector implies, e.g., the digital brokering of physical services such as car repair. At the high end, service automation considers services that can be electronically consumed in wide settings.

Second, and similar to the area of physical products, Standardization [4] is the basis and prerequisite of every further development of a domain. Therefore, standards will play a significant role also in the services industry. Standards are expected to drive the professionalization and industrialization of the service industry, to increase transparency, to lead to higher value services, and eventually to contribute to the overall development of the service economy [1].

With respect to Specialization [6], services once targeted at a specific market, are rebranded and repurposed to fit new consumer needs or other markets in order to extend the reach. Repurposing is facilitated by the automation, since even physical services can be brokered digitally and might be accessed by additional markets and regions.

The ongoing industrialization of the services sector spawned many research activities and even the call for a new discipline, viz., service science [3]. A particular research focus is the systematic use of the Internet for new ways of value creation in the services sector - frequently called the Internet of Services [7]. The basic concepts of the Internet of Services are explained in Section 2 where the need for a standardized service description is elaborated. The latter is represented by the Unified Service Description Language (USDL) (cf. Section 3). Finally, strategic implications are given in Section 4.

\section{The Internet of Services - Basic Concepts}

The basic idea of the Internet of Services is to systematically use the Internet for new ways of value creation in the services sector. There are different angles from 
which one may look at this approach. From an IT perspective, service oriented architectures, software-as-a-service, as well as business process outsourcing are related trends. In this context, the concept of service is referring to a technical understanding of software functions provided as Web services. But services in a broader sense are more than technical capabilities that can be invoked by computer program interfaces. When referring to the importance of the services economy in Section 1, we clearly went beyond the purely technical perspective. Therefore, it is important to clarify what we mean by the term service Here is our proposal for a definition:

Definition 1. A service is a commercial transaction where one party grants temporary access to the resources of another party in order to perform a prescribed function and a related benefit. Resources may be human workforce and skills, technical systems, information, consumables, land and others.

Let us illustrate the definition with an example of a rating service, which defines the creditworthiness of companies. Before selling something to a company, one may want to know how creditworthy it is, so payment conditions can be arranged accordingly. For companies with poor ratings, payment in advance will be asked for while companies with good rating may pay on delivery. The rating service, by nature, is information. As a consequence, the main resources involved are information systems and, most importantly, their content. The temporary access to this information is identical to the service delivery. There is some price scheme to grant the access to this information; examples could be a flat rate or a pay-per-use model. The function is to receive a rating and the benefit to reduce the transaction risk.

\subsection{The Digital Footprint of Services}

The service definition above covers a broad range of services. It is therefore useful to find criteria to differentiate the various kinds of services. One obvious criterion relates to the digital footprint of a service: how and to what degree are information technologies used to instantiate a service.

As an example, think about logistic services. The traditional service is about granting access to some socio-technical resources to ship something from one point to another. With digital media, the information flow associated to the material flow became more and more important. As an example, information about delays in just-in-time delivery is almost as important as the material flow itself. Even without introducing a strict metric, it is intuitive to say that the digital footprint of just-in-time logistics is higher than in traditional transportation.

In the example above, information technologies contribute to the coordination of the core process. But digital media can also add to customer experience. Consider the example of a haircut. It is in itself a manual service. But the service may be enriched by digital media: the appointment may be done over the web, the hair cut may be chosen in advance at home out of a photo gallery or the hair colour may be presented as an overlay on the portrait photo of the customer. 
Another dimension of digital footprint comes from new ways of customer $\mathrm{co}$ creation. E-banking is a good example for that. Here, the customer has web access to the bank's information systems directly over digital media. This digital self service makes the overall process more efficient and provides more transparency to the customer. Part of the service delivery is shifted to the service consumer, but creates value for both service consumer and service provider.

In general, a simple thought experiment can help to illustrate the digital footprint of a service. Just imagine all computer system would shut down for a given time. To what degree would it be possible to deliver the service? If you apply this thought experiment to the services of our daily life, it becomes quite clear that the digital footprint in services is already significant.

Yet, as of today, there is no normative way of describing the services in a unified and machine-readable way. Such a description would wrap a service and would expose it in a novel way. This kind of digital footprint is what we aiming for with the Unified Service Description Language.

\subsection{Complementing the Service Oriented Architecture Paradigm}

For Web services, the SOA paradigm and Web Service Description Language (WSDL) did provide standards for technical service ecosystems. Based on these standards, the promise of SOA was to lower effort of integration of services coming from different information systems. Even if in many practical situations there are still organizational and technical obstacles to fully leverage the potential of $\mathrm{SOA}$, it is clear that the paradigm does indeed offer possibilities to combine and integrate technical services in a faster, more flexible, and more consistent way.

The Internet of Services and the Unified Service Description Language take this approach to the next level. They complement the SOA approach by adding the operational and business aspects to it. For the context of the aforementioned creditworthiness service, it is not only important to define how to technically invoke it (give an address and the interfaces), but it is essential to define the price scheme, the service level agreement, and the terms and conditions when consuming the service and paying for it.

There is another extension of the SOA paradigm. It relates to the roles that come into play in service networks. In SOA, three basic roles have been defined: the service provider, the service consumer and the service mediator or broker. In addition to these roles, we follow the proposal of [2] to also consider the service hoster, gateway, aggregator, and channel maker. The service hoster is an example for an intermediary that catalogues special types of services, namely infrastructure-as-a-service and platform-as-a-service offerings (commonly termed cloud-computing services). It also provides means to interface uniformly with the providers of these services, i.e., re-hosts services through cloud computing environments. Likewise, the service gateway is a specific intermediary that provides interoperability through cataloguing and interfacing with a choice of a 3rd Party B2B gateway, which provides services such as message translation and store-forward processing. The service aggregator provides additional value 
by packaging and combining services. Finally, the service channel maker is positioned at the consumer end of the service provisioning chain where services are channeled into user environments and consumed. Other roles may emerge when service networks establish, e.g., a clearinghouse role. What is important in our context is the fact that with the many roles involved in the Internet of Services, the need for a common language for the description of operational and commercial metadata becomes important.

\section{The Unified Service Description Language}

As pointed out previously, the Internet of Services wants to make services tradable in digital media. In particular, with USDL, we want to offer a language that creates a commercial envelope around services. Technical services may be lifted to business services, while the same language should also be able to describe rather manual or physical services. As many services have a hybrid character with both, a digital and physical or manual footprint, a unified service description language can facilitate the combination and aggregation of such services.

The Unified Service Description Language (USDL) is proposed as a normative and comprehensive master data model for commercial meta data of IT, physical, or hybrid services. More specifically, USDL allows for a unified description of business, operational and technical aspects of services (see Fig. 1). USDL aims at a holistic service description putting special focus on business aspects such as ownership and provisioning, release stages in a service network, composition and bundling, pricing and legal aspects among others, in addition to technical aspects. It proposes a consolidated foundation for service-based systems enabling different roles to participate in diverse aspects of provisioning in service networks.

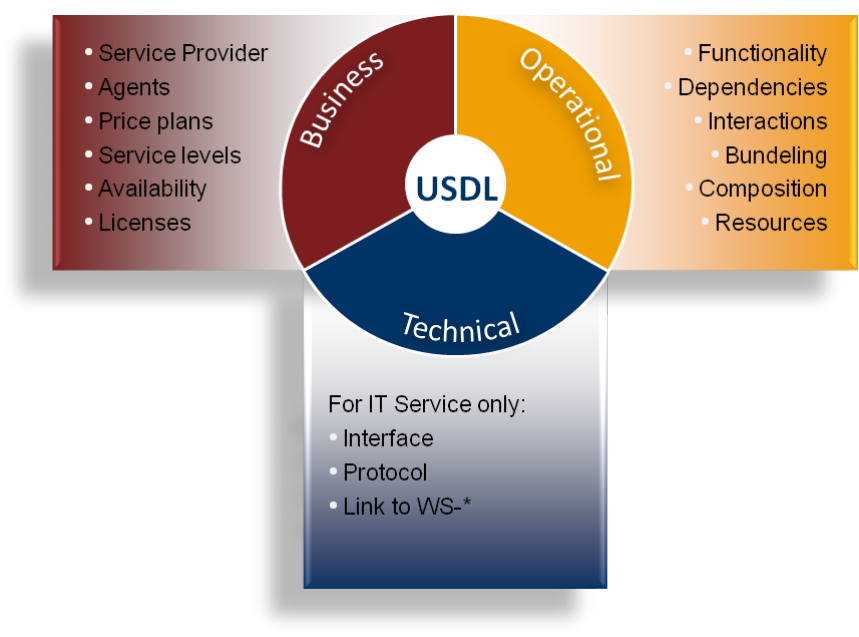

Fig. 1. USDL unifies business, operational, and technical master data of a service. 
Technically speaking, USDL was created using the Eclipse Modeling Framework (EMF) because it was aligned with the remaining landscape and it allows for easy tool creation. Based on the EMF models, XML schemas (XSD) were created for XML-based serialization (https://sourceforge.net/projects/usdlmodel/). We currently work also on a Linked Data representation.

\section{Conclusion}

We anticipate that the Internet of Services and USDL will help to unlock the long tail in service networks. We think that both for the business area and consumers this will lead to a wide range of relevant new services. They will create tangible benefits for their users and economic growth with new value propositions.

The need for standardization becomes apparent when considering the uptake of service networks in the Internet. If participants in a service network specialize to play a specific role in provisioning and delivering services, they act as intermediaries between other participants in the network. They need to disclose, exchange, and comprehend business information about services (pricing, terms and conditions, service-level agreements, etc.) in a standardized way. A standardized and machine readable description will facilitate interoperability between such roles on the business level. The recently closed USDL Incubator Group (http://www.w3.org/2005/Incubator/usdl/) confirmed this need for standardization, since many companies and individuals joined and contributed.

\section{References}

1. Grundstruktur für die Beschreibung von Dienstleistungen in der Ausschreibungsphase. PAS 1018:2002-12, Deutsches Institut für Normung (DIN), Beuth Verlag, Berlin, 2002.

2. Alistair Barros and Uwe Kylau. Service Delivery Framework - An Architectural Strategy for Next-Generation Service Delivery in Business Network. In Patrick Kellenberger, editor, Proceedings 2011 Annual SRII Global Conference SRII 2011, 30 March - 2 April 2011, San Jose, California, USA, pages 47-58. IEEE Computer Society Conference Publishing Services (CPS), 2011.

3. Henry Chesbrough and Jim Spohrer. A research manifesto for services science. Commun. ACM, 49(7):35-40, 2006.

4. Paul A. David and W. Edward Steinmueller. Economics of compatibility standards and competition in telecommunication networks. Information Economics and Policy, 6:217-241, 1994.

5. Silvia Krings, editor. Statistisches Jahrbuch 2010 für die Bundesrepublik Deutschland mit Internationalen Übersichten. Statistisches Bundesamt, Wiesbaden, Germany, 2010.

6. G.B. Richardson. The organisation of industry. Economic Journal, 82(327):883-896, 1972.

7. Orestis Terzidis, Daniel Oberle, Andreas Friesen, Christian Janiesch, and Alistair Barros. The Internet of Services and USDL. In Alistair Barros and Daniel Oberle, editors, Handbook of Service Description: USDL and its Methods, chapter 1, pages 1-16. Springer, New York, 2011. 